\title{
The Effects of Silver, Zinc Oxide, and Titanium Dioxide Nanoparticles Used as Dentin Pretreatments on the Microshear Bond Strength of a Conventional Glass lonomer Cement to Dentin
}

This article was published in the following Dove Press journal:

International Journal of Nanomedicine

\author{
Zahra Jowkar (D) \\ Zahra Fattah (iD) \\ Saeedreza Ghanbarian ${ }^{2}$ \\ Fereshteh Shafiei ${ }^{1}$ \\ 'Oral and Dental Disease Research \\ Center, Department of Operative \\ Dentistry, School of Dentistry, Shiraz \\ University of Medical Sciences, Shiraz, \\ Iran; ${ }^{2}$ Department of Operative \\ Dentistry, School of Dentistry, Shiraz \\ University of Medical Sciences, Shiraz, \\ Iran
}

\begin{abstract}
Aim: This study was conducted to evaluate the effects of three nanoparticle solutions used as dentin pretreatments on the microshear bond strength ( $\mu$ SBS) of a conventional glass ionomer cement (GIC) to dentin.

Materials and Methods: Ninety intact human molars were used after sectioning their occlusal surfaces to expose flat dentin surfaces. The specimens were randomly assigned to nine groups $(\mathrm{n}=10)$. Group A was the control group (without using the cavity disinfectant). In groups $\mathrm{B}, \mathrm{C}, \mathrm{D}$, and $\mathrm{E}$, the prepared dentin surfaces were treated with $1 \mathrm{cc} 2 \%$ chlorhexidine (CHX), $0.1 \%$ silver nanoparticle (SNP), $0.1 \%$ titanium dioxide nanoparticle (TNP), and $0.1 \%$ zinc oxide nanoparticle (ZNP) solutions for 1 minute, respectively, before applying the conditioner. CHX, SNPs, TNPs, and ZNPs were applied for 1 minute after applying the conditioner in groups F, G, H, and I, respectively. The specimens were restored with a conventional GIC and underwent $\mu$ SBS testing after 24 hours. The data were analyzed using the one-way analysis of variance and Tukey's test $(\mathrm{p}=0.05)$.

Results: The applications of the nanoparticles (SNP, TNP, and ZNP) after the conditioner were associated with significantly greater $\mu \mathrm{SBS}$ values compared to that of the control group ( $p$ values $<0.05$ ). Significantly higher $\mu$ SBS values were observed when TNP or ZNP was applied after the conditioner compared to their applications before the conditioner ( $p$ values $<0.05$ ). The highest $\mu$ SBS values were observed when TNP was applied after the conditioner.
\end{abstract}

Conclusion: Dentin pretreatment with the nanoparticles after applying the conditioner enhanced the bond strength of the GIC to dentin compared with the control group. The best results were obtained for the TNPs applied after the conditioner.

Keywords: glass ionomer cement, microshear bond strength, nanoparticles

\section{Introduction}

A common and widely accepted dental restorative material is glass-ionomer cement (GIC). GIC offers unique features such as good biocompatibility, chemical bonding to enamel and dentin, low thermal expansion coefficient, and anti-cariogenic properties due to the release of fluoride. ${ }^{1}$ Although GICs have antibacterial properties due to the release of fluoride, the main reported reason for their failure is secondary caries which might indicate the insufficient antibacterial effect of GICs. ${ }^{2}$ Besides, after the removal of the infected caries and leaving the caries-affected tissue based on
Correspondence: Fereshteh Shafiei

Tel +98-7l-36263193

Fax +98-7I-36280804

Email shafief@sums.ac.ir 
a minimally invasive technique which is advocated for tooth preparation, the bacteria might still be present on the prepared tooth surfaces. ${ }^{3}$ The longevity of the restoration might also be jeopardized if bacterial invasion and microleakage occur at the tooth-restoration interface during clinical service due to the micro gaps that might form there. ${ }^{4}$ To combat bacterial invasion and growth and to reduce sensitivity and the potential development of residual caries, two main strategies have been suggested. The first one is to incorporate different antibacterial agents into the restorative materials and the second one is to pretreat the prepared tooth walls with a cavity disinfectant before restoration placement. ${ }^{1,5,6}$ A common cavity disinfectant is chlorhexidine (CHX). However, CHX could not offer a durable antibacterial efficacy due to its solubility and loose electrostatic bonds. ${ }^{7}$

Recently, the application of nanoparticles (NPs) such as metal NPs has become an important area of research in dentistry mainly because of their unique properties such as their size of less than $100 \mathrm{~nm}$, large proportion of surface atoms, large surface area, and high surface energy. ${ }^{8}$ Additionally, bacterial resistance is less likely to develop in metal NPs compared to the majority of commercially available antibiotics. ${ }^{9}$ Three nanoparticles that have previously been used in the field of dentistry are silver nanoparticles (SNPs), zinc oxide nanoparticles (ZNP), and titanium dioxide nanoparticles (TNPs). ${ }^{6,10}$

The biocompatibility of the SNPs especially in lower concentrations has been previously confirmed. ${ }^{11}$ Moreover, SNPs have demonstrated broad-spectrum and long-term antibacterial and antiviral activities even in low concentrations. ${ }^{11,12}$ The higher antibacterial effect of SNPs (25-fold) compared to that of CHX in a similar concentration has also been demonstrated. ${ }^{13}$ ZNPs have confirmed antibacterial effects against different types of dental plaque bacteria including Lactobacillus and S. mutans. ${ }^{14}$ The selective toxicity of ZNPs against bacteria with minimal effects on human cells has been previously demonstrated. ${ }^{15}$ TNPs possess a potent antibacterial effect, pleasing color, and high biocompatibility. In addition, they have a higher bactericidal effect than chlorhexidine. ${ }^{13,16,17}$ Moreover, TNPs have been suggested to be used as reinforcing fillers in GICs. ${ }^{17}$ It has been reported that the incorporation of TNPs into GICs has resulted in an improved antibacterial activity and some physical properties including microhardness, flexural strength, and compressive strength. ${ }^{1}$ When used as enamel and dentin pretreatments before composite bonding procedures, SNPs, TNPs, and ZNPs did not show any adverse effects on the bond strength values of the adhesive system and the SNPs demonstrated the best results. ${ }^{6}$

No previous study has been conducted on the effect of dentin pretreatment with nanoparticles on the bond strength of conventional GIC to dentin. Therefore, the purpose of this study was to investigate the effects of SNPs, TNPs, and ZNPs used as dentin pretreatments on the microshear bond strength ( $\mu \mathrm{SBS})$ of a conventional GIC to dentin.

\section{Materials and Methods Specimen Preparation}

After the study design was approved by the Research and Ethics Committee of Shiraz University of Medical Sciences (Protocol \# IR.SUMS.DENTAl.REC.1398.41), ninety caries-free human third molars were collected for this experimental study. A blinded calibrated operator performed all of the procedures of this study. The teeth were cleaned with a periodontal curette and stored in a $0.5 \%$ chloramine solution at $4^{\circ} \mathrm{C}$ for less than 4 weeks after extraction. The roots of the teeth were removed at the cementoenamel junction using a water-cooled low-speed cutting machine (Mecatome T201 A, Presi, Grenoble, France). The occlusal thirds of the crowns including occlusal enamel and the superficial dentin were removed using the water-cooled low-speed cutting machine perpendicular to the long axis of the teeth to obtain flat midcoronal dentin surfaces. The sectioned teeth were embedded in acrylic resin blocks (Acropars, Marlik Co., Tehran, Iran) with the cut dentin surfaces oriented perpendicular to the bottom of the molds. After creating a uniform smear layer by slightly wet-grinding the cut dentin surfaces with 320-grit silicon carbide papers for 1 minute, the dentin surfaces were rinsed and dried with an air-water syringe.

\section{Dentin Surface Pretreatment}

The specimens were assigned into nine experimental groups $(\mathrm{n}=10)$ based on the dentin pretreatments with a $2 \% \mathrm{CHX}$ solution (Consepsis, Ultradent Inc., South Jordan, UT, USA) or three different NPs (SNPs, TNPs, and ZNPs; purchased from ASEPE Company, Tabriz, Iran). The SNPs, TNPs, and ZNPs had the particle sizes of $20 \mathrm{~nm}$, $<50 \mathrm{~nm}$, and $20 \mathrm{~nm}$, respectively.

In group A (control group), a cavity conditioner (Cavity Conditioner, GC Co, Japan) was applied on the prepared dentin surfaces according to the manufacturer's instructions. $1 \mathrm{~mL}$ of a $2 \% \mathrm{CHX}$ solution was applied on 
the prepared dentin surfaces using a foam pellet saturated with the solution for 1 minute. Then, the dentin surfaces were blot-dried to remove the excess $\mathrm{CHX}$ before applying the conditioner in group B and after applying the conditioner in group $\mathrm{F}$. In groups $\mathrm{C}, \mathrm{D}$, and $\mathrm{E}$, the prepared dentin surfaces were pretreated with 1 cc $0.1 \%$ SNP, $0.1 \%$ TNP, and $0.1 \%$ ZNP solutions, respectively, for 1 minute, before applying the conditioner. Then, the dentin surfaces were rinsed thoroughly for 1 minute. SNPs, TNPs, and ZNPs were applied for 1 minute after applying the conditioner in groups $\mathrm{G}, \mathrm{H}$, and I, respectively. Then, the dentin surfaces were rinsed thoroughly for 1 minute.

\section{Microshear Bond Strength Testing ( $\mu$ SBS)}

A hollow cylinder with an approximate height of $0.5 \mathrm{~mm}$ and an internal diameter of $0.7 \mathrm{~mm}$ that was cut from microbore Tygon tubing (R-3603, Norton Performance Plastic, Cleveland, $\mathrm{OH}$ ) was placed on the prepared dentin surface defined by an adhesive tape with a punched hole over the center of the flattened dentin surface in each sample. A conventional GIC (GC Fuji II, GC Corporation, Tokyo, Japan) was used to fill the hollow cylinder placed on the dentin surfaces according to the manufacturer's instructions. The samples were stored in water at $37^{\circ} \mathrm{C}$ for 24 hours for complete setting. The Tygon tubing was carefully removed from the GIC with a scalpel. The samples were subjected to a shear force with a direction parallel to the bonded interface in a universal testing machine at the cross-head speed of $1 \mathrm{~mm} / \mathrm{min}(\mathrm{MPa})$ until failure occurred. The recorded load at failure was divided by the bonded surface area to measure the $\mu \mathrm{SBS}$ in $\mathrm{MPa}$ for each sample.

\section{Failure Mode Analysis Under the Stereomicroscope}

The debonded interface was examined under a stereomicroscope (Carl Zeiss Inc., Oberkochen, Germany) at $\times$ 40 to determine the failure mode for each sample. The observed failure modes were classified as follows: (A) adhesive failure within the adhesive interfacial zone $(>75 \%$ failure between the GIC and the dentin); (B) cohesive failure in the GIC or the dentin ( $>75 \%$ failure in the GIC or the dentin); and (C), mixed adhesive failure and cohesive failure. ${ }^{18}$

\section{Statistical Analysis}

The Kolmogorov-Smirnov test was used to assess the normality of the data. The mean $\mu$ SBS values ( \pm standard deviations) of the experimental groups were subjected to the one-way analysis of variance (ANOVA) and multiple comparisons of Tukey's test. The data analyses were performed using SPSS software version 17 (SPSS Inc., Chicago, USA) $(\mathrm{p}<0.05)$.

\section{Results}

The mean $\mu \mathrm{SBS}$ values and the standard deviations (SD) in $\mathrm{MPa}$ are presented in Table 1. The one-way ANOVA showed that there are significant differences in the mean $\mu \mathrm{SBS}$ values among the nine experimental groups $(p=0.001)$. Tukey's test was performed for pair-wise comparisons.

The mean $\mu$ SBS values of the three groups in which the nanoparticles were applied after the conditioner were statistically significantly higher than that of the control group ( $\mathrm{p}$ values $<0.05$ ). The highest mean $\mu$ SBS was observed when TNP was applied after the conditioner ( $p$ values $<0.05$ ). However, no statistically significant difference was observed between the $\mu$ SBS of the group pretreated with TNP after conditioner application and that of the group pretreated with ZNP after conditioner application ( $\mathrm{p}$ value $>0.05)$.

Significantly higher $\mu$ SBS values were observed when TNP or ZNP were applied after the conditioner compared to their applications before the conditioner ( $p$ values $<0.05$ ). The mean $\mu$ SBS of the group pretreated with SNP after conditioner application was higher than that of the group pretreated with SNP before conditioner application, albeit the difference was not statistically significant.

Table I The Means and Standard Deviations of the Microshear Bond Strength ( $\mu \mathrm{SBS}$ ) to Dentin of the Experimental Groups

\begin{tabular}{|l|l|l|}
\hline Group & Experimental Condition & $\begin{array}{l}\text { Microshear Bond } \\
\text { Strength ( } \boldsymbol{\mu S B S} \text { ) } \\
\text { to } \\
\text { Dentin }\end{array}$ \\
\hline A & Control (no pretreatment) & $2.17 \pm 0.61$ \\
B & CHX (applied before & $2.32 \pm 0.97$ \\
& conditioner) & \\
C & SNP (applied before conditioner) & $2.59 \pm 0.32$ \\
D & TNP (applied before conditioner) & $2.21 \pm 0.47$ \\
E & ZNP (applied before conditioner) & $2.09 \pm 0.61$ \\
F & CHX (applied after conditioner) & $3.05 \pm 0.55$ \\
G & SNP (applied after conditioner) & $3.24 \pm 0.50$ \\
H & TNP (applied after conditioner) & $4.81 \pm 0.98$ \\
I & ZNP (applied after conditioner) & $4.07 \pm 1.22$ \\
\hline
\end{tabular}

Abbreviations: $\mathrm{CHX}$, chlorhexidine; SNP, silver nanoparticle; TNP, titanium dioxide nanoparticle; ZNP, zinc oxide nanoparticle. 
Table 2 The Failure Modes of the Experimental Groups

\begin{tabular}{|l|l|l|l|l|}
\hline \multirow{2}{*}{ Group } & \multirow{2}{*}{ Experimental Condition } & \multicolumn{2}{|l|}{ Failure Mode } \\
\cline { 3 - 5 } & & Adhesive & Cohesive & Mixed \\
\cline { 3 - 5 } & & 2 & 0 & 8 \\
B & Control (no pretreatment) & 2 & 0 & 9 \\
C & CHX (applied before conditioner) & 1 & 0 & 8 \\
D & SNP (applied before conditioner) & 2 & 0 & 9 \\
E & TNP (applied before conditioner) & 1 & 0 & 7 \\
F & ZNP (applied before conditioner) & 3 & 0 & 8 \\
G & CHX (applied after conditioner) & 2 & 0 & 7 \\
H & SNP (applied after conditioner) & 3 & 0 & 8 \\
I & TNP (applied after conditioner) & 2 & 0 & 9 \\
\hline
\end{tabular}

Abbreviations: $\mathrm{CHX}$, chlorhexidine; SNP, silver nanoparticle; TNP, titanium dioxide nanoparticle; ZNP, zinc oxide nanoparticle.

The application of CHX after the conditioner resulted in a statistically significantly lower mean $\mu$ SBS compared to when TNP was applied after the conditioner $(\mathrm{p}<0.05)$. No other statistically significant differences were found between the group in which CHX was applied after the conditioner and the other experimental groups ( $p$ values $>0.05$ ).

The mean $\mu$ SBS value of the group in which ZNP was applied after the conditioner was statistically significantly higher than those of the groups in which SNP or TNP was applied before the conditioner ( $\mathrm{p}$ values $<0.05$ ). The application of SNP after the conditioner resulted in a significantly higher $\mu$ SBS compared to when TNP or ZNP was applied before the conditioner ( $\mathrm{p}$ values $<0.05$ ).

The failure mode analysis showed that adhesive failure was the most prevalent failure mode in all the study groups (Table 2).

\section{Discussion}

This study was conducted to evaluate the effects of pretreatment with CHX, SNPs, TNPs, and ZNPs on the $\mu$ SBS of a conventional GIC to dentin. According to the results of this study, none of the nanoparticle solutions used in this study interfered with the $\mu$ SBS of GIC to dentin. Moreover, after applying the conditioner, the three groups pretreated with nanoparticles demonstrated higher $\mu$ SBS values than the control group. The best result was obtained when TNP was applied after the conditioner.

The $\mu$ SBS test which is a facile and reliable method has been utilized in this study to assess the bond strength of GIC to dentin. Due to the small areas which are tested in the $\mu \mathrm{SBS}$ test, multiple specimens can be prepared from the same tooth. This test does not have the drawbacks of the macroshear test including the mixed loading mode, the inhomogeneous distribution of stress in the loaded cross- section, and the tendency of cohesive failure in the dentin substrate instead of failure at the dentin/restorative material interface at loads far less than the shear strength. ${ }^{19}$ In this study, the $\mu$ SBS test was performed 24 hours after the initial setting of GIC to allow the complete setting of GIC during this period.

Different cavity disinfectants have been utilized previously. ${ }^{20}$ An ideal cavity disinfectant should not compromise the bond strength value of the restorative material to the tooth structure. ${ }^{19}$ Therefore, the effects of CHX and the nanoparticles used as cavity disinfectants before restoration placement on the $\mu$ SBS of GIC to dentin were assessed in this study.

CHX was used in this study for dentin pretreatment before restoration placement. CHX is a common cavity disinfectant which demonstrates a matrix metalloproteinase (MMP)-inhibitory effect and therefore can reduce the loss of bond strength over time without completely preventing it. ${ }^{21}$ However, some concerns have been mentioned for CHX. It is not completely clear whether the MMP-inhibitory effect of CHX is temporary or permanent. Moreover, CHX does not hinder water sorption and hydrolysis. ${ }^{22}$ Other concerns regarding CHX used as a cavity disinfectant are its possible negative effects on the bond strength and whether it can have a long-term antibacterial effect and remove the viable microorganisms completely. ${ }^{20,23}$ Therefore, efforts are underway to find alternative dentin pretreatment agents to be used as cavity disinfectants. In this study, the application of CHX did not affect the bond strength of GIC to dentin compared to the control group which was in line with the findings of a previous study. ${ }^{24}$ Moreover, in this study, the $\mu$ SBS of the group in which CHX was applied after the conditioner was more than that of the group in which CHX was applied before the conditioner, albeit the difference was not statistically significant.

Due to the antibacterial, antiviral, anti-adhesive, and anti-inflammatory properties of NPs and their effects on controlling biofilm formation within the oral cavity, the application of nanomaterials has attracted increasing interest from researchers in dentistry. ${ }^{10,25}$ To benefit from the antibacterial properties of NPs in dentistry, two strategies have been investigated including adding NPs to the dental materials and coating the surfaces with NPs to prevent the adhesion of bacteria. ${ }^{26}$ The second strategy was used in this study to benefit from the antibacterial properties of the NPs and three types of nanoparticle solutions (SNP, ZNP, and TNP) were used as dentin pretreatments. 
Silver has been used in the field of dentistry as an important broad-spectrum antibacterial agent with low toxicity and good biocompatibility especially in low concentrations. ${ }^{9}$ Multiple antibacterial mechanisms have been proposed for SNPs such as increased permeability and disrupting the integrity of bacterial cell membranes due to the adherence and penetration of silver into the bacterial cell wall, inactivating the vital enzymes of bacteria, and inhibiting the DNA replication ability of bacteria. ${ }^{12}$ When used as an antimicrobial irrigating solution in the preparation of the root canal for endodontic treatment, the SNP solution was able to prevent biofilm formation and reduce bacterial adhesion. ${ }^{11}$ Moreover, silver nanoparticles have demonstrated a much higher antibacterial activity $(25 \mathrm{~nm})$ than zinc oxide $(125 \mathrm{~nm})$ and gold $(80 \mathrm{~nm})$ nanoparticles. ${ }^{27}$ Silver can interact with oxide, phosphate, chloride, and proteins and form a silver compound with a relatively low solubility within dentinal tubules. ${ }^{28}$ This might potentially lead to a durable and gradual release of silver ions and thus to the long-term antibacterial efficacy of silver at the tooth-restoration interface. ${ }^{28}$ According to the results of this study, when applied before the conditioner, SNP did not negatively affect the $\mu$ SBS of GIC to dentin compared with the control group or the group in which CHX was applied before the conditioner. Moreover, when SNP was applied after the conditioner, a higher $\mu$ SBS was obtained compared to that of the control group.

Another nanoparticle which was applied as dentin pretreatment in this study was ZNP. Because of the increased surface-to-volume ratio of nanoparticles, ZNPs possess an improved antibacterial activity compared to zinc oxide. ${ }^{29}$ Two proposed mechanisms for the antibacterial properties of ZNPs are the modification of the bacterial cell membrane activity and the generation of active oxygen species such as $\mathrm{H} 2 \mathrm{O} 2$ that inhibit bacterial growth. ${ }^{30}$ The application of zinc oxide could inhibit the demineralization of dentin, decrease decalcification which occurs following orthodontic treatment, and stimulate the remineralization of hard tissue. ${ }^{31,32}$ ZNPs inhibit the action of MMPs which participate in the degradation of dentin collagen. ${ }^{33}$ Compared to that of CHX, a much longer inhibitory effect on collagen degradation in demineralized dentin has been previously reported for zinc oxide. ${ }^{34}$ The results of this study demonstrated that the application of ZNP before the conditioner did not adversely affect the $\mu$ SBS of GIC to dentin compared to the control group and the groups in which CHX was used. Additionally, when applied after the conditioner, ZPS increased the $\mu$ SBS compared to the control group and the group in which CHX was applied after the conditioner.

TNPs were also used as a dentin pretreatment in this study. TNPs have a high surface energy and significant antibacterial activity by producing free radicals which cause oxidative damage in the cell walls of microorganisms. They also have good antiadhesive properties against Streptococcus mutans. ${ }^{35}$ Moreover, TNPs have demonstrated better antibacterial properties than CHX. ${ }^{9}$ TNPs have been proposed for preventing white spot formation during orthodontic treatment. ${ }^{9}$ In the current study, the best result was obtained when TNP was used after the conditioner. Moreover, dentin pretreatment with TNP after applying the conditioner resulted in a higher $\mu$ SBS compared to when TNP was applied before the conditioner.

When used as dentin pretreatments, SNPs, TNPs, and ZNPs had no adverse effects on the bond strengths of resin composite and resin cements to coronal and radicular dentins, respectively. ${ }^{6,36}$ Moreover, SNP pretreatment demonstrated a higher coronal dentin bond strength value compared with ZNP and TNP pretreatments. ${ }^{6}$ According to the results of this study, none of the three used nanoparticles adversely affected the $\mu$ SBS of GIC to dentin compared to the control group or the groups pretreated with CHX. The application of the nanoparticles after the conditioner significantly increased the $\mu$ SBS of GIC to dentin compared to the control group. Moreover, dentin pretreatment with the nanoparticles applied after the use of a conditioner significantly increased the $\mu$ SBS of GIC to dentin compared to their application before using the conditioner. Generally, the application of the conditioner could provide some micro-retention at dentin, ideally rendering the dentin surface receptive to chemical bonding. ${ }^{37}$ In this study, the higher $\mu$ SBS values of the groups in which the nanoparticles were applied after the conditioner compared with those in which the nanoparticles were applied before the conditioner might be attributed to the effects of conditioner application on the dentin substrate. These include the removal of the smear layer and surface contaminants, alteration of the surface free energy and the wettability of dentin, partial demineralization of the dentin surface, and the exposure of the dentin collagen network which might result in the close adaptation of the nanoparticles to dentin surfaces and the better diffusion of these small size molecules into a partially demineralized tooth structure. ${ }^{24,37}$ Nanoparticles diffused between partially demineralized collagen fibers might increase the surface 
free energy previously reduced by the partial demineralization of dentin resulting from applying the conditioner. ${ }^{37}$ However, the effects of the nanoparticles on the free energy and wettability of the dentin surface were not evaluated in this study and should be investigated in the future.

One of the unique properties of GIC is its chemical bonding to the tooth structure which is formed by the reaction of the carboxylate groups in polyacrylic acid with the phosphate ions in the dental tissue. ${ }^{38}$ The properties of the dental substrate could affect the bond strength of GICs to the tooth structure. ${ }^{38}$ Dentin bonding is challenging due to the heterogeneous structure of the dentin consisting of a complex organic structure with a low surface energy and an inorganic structure. ${ }^{39}$ The findings of this study showed that dentin pretreatments with NPs not only did not compromise the bonding ability of GIC to dentin but also were able to influence the $\mu$ SBS of GIC to dentin positively especially when applied after the conditioner. This finding might be due to the possible altered surface energy and wettability of the dentin substrate following nanoparticle application which should be assessed in future studies. Moreover, the water-based characteristics of the nanoparticles used in this study might play a potential role in changing the surface tension of the dentin substrate and making it more receptive to the two-fold micromechanical and chemical bonding mechanism of GIC to dentin.

Among the nanoparticles used in this study, the best result belonged to TNP applied after the conditioner. For the time being, the authors cannot give any clear logical explanation for this finding. However, this finding might be related to the differences in the size and surface-tovolume ratio of the applied nanoparticles, their morphologies, chemical and colloidal stability, surface free energy, and aggregation stability which lead to their different interactions with the tooth structure and the restorative material. More studies are needed to assess the exact effects of the mentioned factors on the bond strength properties of the nanoparticles to the tooth structure.

The present study has some limitations. The antibacterial effects of the three nanoparticles used in this study were not compared with each other. More studies are needed in the future to compare the antibacterial effects of different nanoparticles against cariogenic bacteria. The influence of aging (mechanical aging or thermal cycling) on the $\mu$ SBS of the GIC to dentin was not assessed in this study. Future studies should also consider the effects of low intermittent functional forces from different directions and temperature changes in the oral environment on the $\mu \mathrm{SBS} .{ }^{40}$ Complex forces resulting from parafunctional habits might also affect the bond strength of the restorative material to the tooth structure which should be considered in future studies. ${ }^{41}$ Additionally, one conventional GIC was assessed in the present study, therefore, the obtained results cannot be generalized to other types of GICs. The long-term antibacterial and bond strength properties of NPs and their probable release into the saliva and oral cavity should also be investigated in the future.

\section{Conclusion}

According to the results of this study, using nanoparticles after the conditioner increased the $\mu$ SBS values of the conventional GIC to dentin and would be beneficial in clinical practice because of their antibacterial properties. The best result was obtained for TNP applied after the conditioner.

\section{Acknowledgments}

The authors thank the Vice-Chancellery of Research, Shiraz University of Medical Sciences for supporting this research and Biomaterial Research Center of Shiraz University of Medical Sciences for testing the specimens (Number: 9801-03-19819). This article is based on Dr. Saeedreza Ghanbarian's dissertation. The authors thank Dr. Mehrdad Vossoughi from the Dental Research Development Center for the statistical analyses. The authors also thank Dr. Arash Moradi for editorial assistance.

\section{Disclosure}

The authors report no conflicts of interest in this work.

\section{References}

1. Garcia-Contreras R, Scougall-Vilchis RJ, Contreras-Bulnes R, Sakagami H, Morales-Luckie RA, Nakajima H. Mechanical, antibacterial and bond strength properties of nano-titanium-enriched glass ionomer cement. J Appl Oral Sci. 2015;23(3):321-328. doi:10.1590/ 1678-775720140496

2. Xie D, Weng Y, Guo X, Zhao J, Gregory RL, Zheng C. Preparation and evaluation of a novel glass-ionomer cement with antibacterial functions. Dent Mater. 2011;27(5):487-496. doi:10.1016/j.dental.2011.02.006

3. Doozandeh M, Firouzmandi M, Mirmohammadi M. The simultaneous effect of extended etching time and casein phosphopeptide-amorphous calcium phosphate containing paste application on shear bond strength of etch-and-rinse adhesive to caries-affected dentin. J Contemp Dent Pract. 2015;16(10):794-799. doi:10.5005/jp-journals-10024-1759

4. Kasraei S, Sami L, Hendi S, AliKhani M-Y, Rezaei-Soufi L, Khamverdi Z. Antibacterial properties of composite resins incorporating silver and zinc oxide nanoparticles on Streptococcus mutans and Lactobacillus. Restor Dent Endod. 2014;39(2):109-114. 
5. Jowkar Z, Jowkar M, Shafiei F. Mechanical and dentin bond strength properties of the nanosilver enriched glass ionomer cement. J Clin Exp Dent. 2019;11(3):e275-e281.

6. Jowkar Z, Farpour N, Koohpeima F, Mokhtari M, Shafiei F. Effect of silver nanoparticles, zinc oxide nanoparticles and titanium dioxide nanoparticles on microshear bond strength to enamel and dentin. J Contemp Dent Pract. 2018;19(11):1404-1411.

7. Ribeiro LGM, Hashizume LN, Maltz M. The effect of different formulations of chlorhexidine in reducing levels of mutans streptococci in the oral cavity: a systematic review of the literature. J Dent. 2007;35(5):359-370

8. Li X, Cui R, Liu W, et al. The use of nanoscaled fibers or tubes to improve biocompatibility and bioactivity of biomedical materials. J Nanomater. 2013;2013:1-16.

9. Borzabadi-Farahani A, Borzabadi E, Lynch E. Nanoparticles in orthodontics, a review of antimicrobial and anti-caries applications. Acta Odontol Scand. 2014;72(6):413-417.

10. Jowkar Z, Hamidi SA, Shafiei F, Ghahramani Y. The effect of silver, zinc oxide, and titanium dioxide nanoparticles used as final irrigation solutions on the fracture resistance of root-filled teeth. Clin Cosmet Investig Dent. 2020;12:141-148. doi:10.2147/CCIDE.S253251

11. Gomes-Filho JE, Silva FO, Watanabe $\mathrm{S}$, et al. Tissue reaction to silver nanoparticles dispersion as an alternative irrigating solution. J Endod. 2010;36(10):1698-1702. doi:10.1016/j.joen.2010.07.007

12. Rai M, Deshmukh S, Ingle A, Gade A. Silver nanoparticles: the powerful nanoweapon against multidrug-resistant bacteria. J Appl Microbiol. 2012;112(5):841-852. doi:10.1111/j.1365-2672.2012.05253.x

13. Besinis A, De Peralta T, Handy RD. The antibacterial effects of silver, titanium dioxide and silica dioxide nanoparticles compared to the dental disinfectant chlorhexidine on Streptococcus mutans using a suite of bioassays. Nanotoxicology. 2014;8(1):1-16. doi:10. 3109/17435390.2012.742935

14. Jones N, Ray B, Ranjit KT, Manna AC. Antibacterial activity of ZnO nanoparticle suspensions on a broad spectrum of microorganisms. FEMS Microbiol Lett. 2008;279(1):71-76. doi:10.1111/j.15746968.2007.01012.x

15. Sharma V, Shukla RK, Saxena N, Parmar D, Das M, Dhawan A. DNA damaging potential of zinc oxide nanoparticles in human epidermal cells. Toxicol Lett. 2009;185(3):211-218. doi:10.1016/j. toxlet.2009.01.008

16. Heravi F, Ramezani M, Poosti M, Hosseini M, Shajiei A, Ahrari F. In vitro cytotoxicity assessment of an orthodontic composite containing titanium-dioxide nano-particles. J Dent Res Dent Clin Dent Prospects. 2013;7(4):192-198. doi:10.5681/joddd.2013.031

17. Elsaka SE, Hamouda IM, Swain MV. Titanium dioxide nanoparticles addition to a conventional glass-ionomer restorative: influence on physical and antibacterial properties. J Dent. 2011;39(9):589-598. doi:10.1016/j.jdent.2011.05.006

18. Kong YY, Chen Y, Shen R, Jiang QZ, Chen ML. Influence of thermocycling on shear bond strength of glass ionomer cement to Er: YAG laser-prepared enamel. Lasers Surg Med. 2016;48 (10):978-984. doi:10.1002/1sm.22452

19. Sadat-Shojai M, Atai M, Nodehi A, Khanlar LN. Hydroxyapatite nanorods as novel fillers for improving the properties of dental adhesives: synthesis and application. Dent Mater. 2010;26 (5):471-482. doi:10.1016/j.dental.2010.01.005

20. Ercan E, Erdemir A, Zorba YO, et al. Effect of different cavity disinfectants on shear bond strength of composite resin to dentin. J Adhes Dent. 2009;11(5):343-346.

21. Rosenthal S, Spångberg L, Safavi K. Chlorhexidine substantivity in root canal dentin. Oral Surg Oral Med Oral Pathol Oral Radiol Endod. 2004;98(4):488-492. doi:10.1016/j.tripleo.2003.07.005

22. Sadek FT, Castellan CS, Braga RR, et al. One-year stability of resindentin bonds created with a hydrophobic ethanol-wet bonding technique. Dent Mater. 2010;26(4):380-386. doi:10.1016/j.dental.2009.12.009
23. Borges FMC, de Melo MAS, Lima JPM, Zanin ICJ, Rodrigues LKA. Antimicrobial effect of chlorhexidine digluconate in dentin: in vitro and in situ study. J Conserv Dent. 2012;15(1):22-26. doi:10.4103/ 0972-0707.92601

24. Aykut-Yetkiner A, Candan U, Ersin N, Eronat C, Belli S, Özcan M. Effect of $2 \%$ chlorhexidine gluconate cavity disinfectant on microtensile bond strength of tooth-coloured restorative materials to sound and caries-affected dentin. $J$ Adhes Sci Technol. 2015;29 (12):1169-1177. doi:10.1080/01694243.2015.1004507

25. Magalhães APR, Santos LB, Lopes LG, et al. Nanosilver application in dental cements. ISRN Nanotechnol. 2012;2012:1-6.

26. Hamouda IM. Current perspectives of nanoparticles in medical and dental biomaterials. J Biomed Res. 2012;26(3):143-151. doi:10.7555/ JBR.26.20120027

27. Hernández-Sierra JF, Ruiz F, Pena DCC, et al. The antimicrobial sensitivity of Streptococcus mutans to nanoparticles of silver, zinc oxide, and gold. Nanomedicine. 2008;4(3):237-240. doi:10.1016/j. nano.2008.04.005

28. Mei ML, Ito L, Cao Y, Li Q, Chu C, Lo EC. The inhibitory effects of silver diamine fluorides on cysteine cathepsins. J Dent. 2014;42 (3):235-329. doi:10.1016/j.jdent.2013.11.018

29. Li F, Weir MD, Chen J, Xu HH. Comparison of quaternary ammonium-containing with nano-silver-containing adhesive in antibacterial properties and cytotoxicity. Dent Mater. 2013;29 (4):450-461. doi:10.1016/j.dental.2013.01.012

30. Xie Y, He Y, Irwin PL, Jin T, Shi X. Antibacterial activity and mechanism of action of zinc oxide nanoparticles against Campylobacter jejuni. Appl Environ Microbiol. 2011;77(7):2325-2331. doi:10.1128/AEM.02149-10

31. Takatsuka T, Tanaka K, Iijima Y. Inhibition of dentine demineralization by zinc oxide: in vitro and in situ studies. Dent Mater. 2005;21 (12):1170-1177. doi:10.1016/j.dental.2005.02.006

32. Spencer CG, Campbell PM, Buschang PH, Cai J, Honeyman AL. Antimicrobial effects of zinc oxide in an orthodontic bonding agent. Angle Orthod. 2009;79(2):317-322. doi:10.2319/011408-19.1

33. Osorio R, Yamauti M, Osorio E, Román JS, Toledano M. Zinc-doped dentin adhesive for collagen protection at the hybrid layer. Eur J Oral Sci. 2011;119(5):401-410. doi:10.1111/j.1600-0722.2011.00853.x

34. Osorio R, Yamauti M, Osorio E, et al. Zinc reduces collagen degradation in demineralized human dentin explants. J Dent. 2011;39 (2):148-153

35. Cao B, Wang Y, Li N, Liu B, Zhang Y. Preparation of an orthodontic bracket coated with an nitrogen-doped TiO2-xNy thin film and examination of its antimicrobial performance. Dent Mater J. 2013;32(2):311-316.

36. Jowkar Z, Omidi Y, Shafiei F. The effect of silver nanoparticles, zinc oxide nanoparticles, and titanium dioxide nanoparticles on the push-out bond strength of fiber posts. J Clin Exp Dent. 2020;12(3): e249-e256.

37. Inoue S, Abe Y, Yoshida Y, et al. Effect of conditioner on bond strength of glass-ionomer adhesive to dentin/enamel with and without smear layer interposition. Oper Dent. 2004;29(6):685-692.

38. Magni E, Ferrari M, Hickel R, Ilie N. Evaluation of the mechanical properties of dental adhesives and glass-ionomer cements. Clin Oral Investig. 2010;14(1):79-87.

39. Lohbauer U. Dental glass ionomer cements as permanent filling materials?-properties, limitations and future trends. Materials. 2010;3(1):76-96.

40. Shafiei F, Saadat M. Micromorphology and bond strength evaluation of adhesive interface of a self-adhering flowable composite resindentin: Effect of surface treatment. Microsc Res Tech. 2016;79 (5):403-407.

41. Safari A, Jowkar Z, Farzin M. Evaluation of the relationship between bruxism and premature occlusal contacts. J Contemp Dent Pract. 2013;14(4):616-621. doi:10.5005/jp-journals-10024-1374 


\section{Publish your work in this journal}

The International Journal of Nanomedicine is an international, peerreviewed journal focusing on the application of nanotechnology in diagnostics, therapeutics, and drug delivery systems throughout the biomedical field. This journal is indexed on PubMed Central, MedLine, CAS, SciSearch ${ }^{\circledR}$, Current Contents ${ }^{\circledR} /$ Clinical Medicine, $^{2}$
Journal Citation Reports/Science Edition, EMBase, Scopus and the Elsevier Bibliographic databases. The manuscript management system is completely online and includes a very quick and fair peer-review system, which is all easy to use. Visit http://www.dovepress.com/ testimonials.php to read real quotes from published authors. 